\title{
Incidencia de la biblioteca pública en los jóvenes
}

\author{
O RLANDA JARAMILlo \\ Profesora de la Escuela Interamericana de Bibliotecología \\ de la Universidad de Antioquia \\ E-mail: ojara@ nutabe.udea.edu.co \\ MóniCa Montoya Ríos \\ Biblioteca Pública Escolar José María Velaz \\ de Fé y Alegría, Regional Bello. Antioquia. \\ E-mail: bjmv@epm.net.co
}

\begin{abstract}
RESUMEN
Considerando la incidencia de la biblioteca como esa huella o marca que que da en losjóvenes luego de usarla, lainvestigación indagó sobre las relacionesy vínculos establecidos entre los programas y servicios que desarrolla la biblioteca pública popular y los jóvenes. Por tanto fue preciso ahondar sobre los usos que hacen de los servicios y de los programas que tiene la biblioteca, sobre la satisfacción de las necesidades de información en el ámbito educativo, recreativo, cultural y organizativo, y sobre las diversas relaciones que se dan entre los jóvenes y la biblioteca, al igual que el grado de satisfacción que estos usos generan.

El artículo se desarrolla a partir de dos ítems: en el primero se presentan los diversos conceptos que se consideran como soportes para argumentarla incidencia: biblioteca pública, jóvenes, servicios y usuarios. El segundo ítem describe la incidencia de la biblioteca en los jóvenes y en él se caracterizan los usos que este grupo poblacional hace de ella y que están en relación directa con los objetivos básicos de la biblioteca pública: informativo, recreativo, cultural, educativo y organizativo.

Palabras clave: Servicios de información; Servicios de información a la comunidad; Necesidades de información; Papel social de las bibliotecas
\end{abstract}

Trabajo recibido el

2 de marzo de 2001 粦

Trabajo aceptado el 5 de mayo de 2001

\section{OUTCOME OF PUBLIC LIBRARIES FOR YOUTH Orlanda Jaramillo y Mónica Montoya-Ríos}

\section{ABSTRACT}

This article forms part of the research " The outcome of public libraries for young people between 15 and 24 years' old from the "Comuna Seis" in the northwest zone of Medellin (Colombia)" carried out in 1999 with the support of the Interamerican School of Library Science of the University of Antioquia. Considering the outcome of a library as the impression or mark it leaves in young people who have experienced its use, the research investigated the relations and ties established between the programs and services developed by the public library and young people. Consequently it was necessary to study 
in-depth the use that is made of the programs and services provided by the library, the satisfaction of information needs in the educational, leisure, cultural and organizational environments, the diverse relationships formed between youth and the library, as well as the degree of satisfaction encountered.

The article develops two main concerns: firstly, the different concepts are presented which lend support to the argument for outcome: public library, youth, services and users. Secondly, the outcome of the library in young people is described, characterizing the use that this sector of the population makes of the library in direct relation to the main objectives of the public library: information, leisure, culture, education and organization.

Key Words: Public Libraries; Information Services to the Community; User Studies; Information Needs; Library Social Roles

\section{INTRODUCCIÓN}

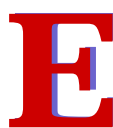

l propósito del documento es caracterizar los usos que los jóvenes hacen de la biblioteca pública y ofrecer un acercamiento a la manera en que interactúa la biblioteca pública con este sector poblacional, así como observar la incidencia que tiene la información de la que se han apropiado en la solución de los problemas cotidianos: de participación, de toma de decisiones, y de identidad y ocio, entre otros. Esto es, describe la huella que deja la biblioteca en los jóvenes que han transitado por ella, e intenta ver si esto da respuesta a sus actividades escolares y académicas, informativas, de ocupación del tiempo libre, de recreación, de agrupación y de encuentro con el otro, para el caso específico de los jóvenes de los sectores populares de Medellín y en el caso de la investigación para los que están ubicados en la Comuna Seis de la Zona Noroccidental. ${ }^{1}$

La investigación permitió constatar la validez de la información, de los programas, y de los servicios y actividades que ofrece la biblioteca pública para solucionar los problemas cotidianos y mostró que la información se convierte en elemento esencial para el desarrollo personal, la participación y la toma de decisiones.

La búsqueda de la incidencia de la biblioteca en los jóvenes se hizo a partir de una investigación cualitativa, apoyada en algunos momentos con técnicas cuantitativas. El enfoque se orientó a describir e interpretar la interacción entre los jóvenes y la biblioteca pública popular, y el uso que éstos hacen de la información en su vida coti-

1 LaZ Zna Noroccidental está ubicada en la ladera occidental del V alle de Aburra, entre el río Medelín y el perímetro urbano occidental. El total delapoblación es de 413.804 habitantes, de los cuales 162.331 son jóvenes. La Comuna Seis es denominada "D oce de octubre" y se caracteriza por ser un territorio con un número considerable de habitantes y con la mayor densidad poblacional: 365 habitantes por hectárea. Ha sido reconocida por el nivel y trayectoria de organización comunitaria que se ha movido desde las juntas de acción comunal, el sindicalismo, el movimiento popular, las organizaciones barriales, las organizaciones juveniles, organizaciones cívicas, comunitarias, culturales, artísticas y proyectos de integración comunal y zonal. D entro de este proceso de organización surgen y se mantienen las bibliotecas populares. 
diana. Se buscaba conocer los significados y las intenciones de las acciones de la biblioteca, de sus funcionarios y de la población juvenil, para lo cual se utilizaron fuentes de información primarias como bibliotecarios, coordinadores de las bibliotecas en estudio, alfabetizadores, jóvenes usuarios escolarizados, jóvenes no escolarizados (habitantes de la calle, de las esquinas y de las canchas de los barrios), líderes comunitarios, profesores y exbibliotecarios. Estas fuentes permitieron caracterizar, identificar y objetivar las relaciones, usos y propuestas -tanto de la biblioteca como de los jóvenes-, en un contexto urbano y en un entorno barrial determinado. Como producto del rastreo de estas fuentes primarias se incluyen algunos testimonios en letra cursiva o en negrilla y entrecomillados, y también se adjuntan algunos gráficos considerados importantes para constatar la incidencia que tuvo la biblioteca en los jóvenes.

Como fuentes de información secundaria se utilizaron investigaciones del área, tesis de grado, textos y documentos consultados en Internet.

\section{DEFINICIÓN DE CONCEPTOS}

Loesnial es queexisteunlugar al quepue deirla getearandbtieneganas deailtivarse odecambiar, arandbtieneganas deser dife rente Algpquela socieadad puedeponer a dis posión dela gete Cræoquehabrá quere pensar a la soieelad comouna epeiede biblictea

Michéle Petit

\section{Biblioteca pública}

Si bien el concepto de biblioteca pública ha evolucionado perfilándose y adaptándose a las dinámicas sociales, ésta sigue siendo en esencia una institución democrática ligada a garantizary desarrollar el derecho a la información, a la educación, a la recreación y a la cultura, aspectos que están estrechamente unidos con la libertad de expresión, base de la convivencia democrática. La biblioteca es un espacio para la formación de la identidad individual y su función está cimentada en la creación de ciudadanos libres que tengan acceso a la información que requiere la toma de decisiones. Por esto la biblioteca pública tiene un gran potencial de intervención en las dinámicas sociales, pues puede contribuir a subsanar las desigualdades económicas, culturales, educativas y organizativas, y equilibrar las posibilidades de desarrollo de las personas.

En concordancia con lo anterior, el concepto de biblioteca pública que se asume en la investigación es el siguiente:

La biblioteca pública es una institución de carácter social (educativa, recreativa, informativa y cultural), financiada y reglamentada por el estado, cuya finalidad es posibilitar el libre acceso a información registrada en un 
soporte documental y que responde a unos criterios de selección y adquisición para la satisfacción de necesidades en el plano educativo, informativo, cultural y de uso del tiempo libre; busca con ello contribuir al mejoramiento de la calidad de vida de todas las personas que hacen parte de una comunidad (municipio, vereda o barrio), parala construcción yarticulación de relaciones democráticas, por medio de servicios y programas gratuitos y coordinados por profesionales del área. ${ }^{2}$

Para que la biblioteca pública cumpla su finalidad o razón de ser en la sociedad, debe asumir los objetivos asignados inicialmente en 1971, por el G rupo de Investigación de Bibliotecas Públicas de la Asociación de Bibliotecarios Británicos, ${ }^{3}$ a saber:

E ducativo: Facilitar y proveer a las personas de materiales y medios para la auto- educación, y posibilitar la apropiación del conocimiento.

* Informativo: O frecer información actualizada, pertinente y de calidad que les permita a las personas solucionar los problemas de la vida diaria, variar el nivel de incertidumbre y establecer bases para un nuevo conocimiento que repercuta en el desarrollo personal y colectivo y genere nuevas motivaciones. Al respecto es pertinente citar la afirmación del bibliotecólogo Luis Bernardo Yepes:

Se requiere una biblioteca para el lector; son múltiples los procedimientos que el hombre contemporáneo encuentra para informarse, pero el menos empleado es el de la palabra escrita. La biblioteca pública debe promover su uso, sólo ella lo puede hacer.4

* Cultural: Convertir el espacio de la biblioteca en centro de la vida cultural del sector, donde se genere, rescate, preserve y difundan losvalores y laidentidad cultural, por medio de la participación activa, del disfrute y de la apreciación de todas las expresiones culturales y artísticas.

* Recreativo: Motivar alas personas para hacer buen uso desu tiempo disponible, con el adecuado suministro de documentos y la realización de programas para el entretenimiento y la recreación.

Los objetivos se materializan en el diseño y desarrollo de servicios y programas que se definen de la siguiente manera:

2 En el caso de labiblioteca pública popular la creación, financiación y reglamentación generalmente corresponde a la comunidad en la cual se inscribe la biblioteca.

Concepto tomado de la investigación : "Incidencia de la biblioteca pública en losjóvenes de 15-24 años de la Comuna Seis, Zona Noroccidental de Medellín”. Medellín : Escuela Interamericana de Bibliotecología, 1999. p. 87.

3 Public Library Research G roup. Public Library aims and objetives. / En: LibraryAssociationRecord No. 73 (Dec. 1971).

4 Luis Bernardo Yepes O. Lemaymisóndelabiblicteca pública Medellín :Comfenalco, 1998. p.9. 


\section{Servicios}

D efinidos como las acciones que se realizan para dar respuesta a las necesidades de información de los usuarios. Son la esencia de la biblioteca, están diseñados para todos los usuarios y tienen permanencia en el tiempo; su diseño involucra cuatro componentes:

* Personal: su actitud y aptitud definen la calidad, eficacia, eficiencia y oportunidad del servicio.

Instalaciones físicas: su bienestar y confort privilegian al usuario.

Tecnologías: facilitan la oportunidad y la eficiencia del servicio.

Procesos: Permiten la eficiencia y eficacia. Adicionan valor a los insumos para transformarlos en servicios, pueden o no hacer uso de la tecnología.

Los servicios requieren:

* Estar respaldados en políticas administrativas que orienten su acción.

* Ser planeados de acuerdo con la finalidad de la biblioteca y las necesidades de información de los usuarios.

* Apoyarse en un manual que oriente sus procedimientos, formas de ejecución y evaluación. ${ }^{5}$

Los servicios que ofrece la biblioteca pública se pueden clasificar en dos categorías: básicos y complementarios:

\section{Servicios básicos:}

A quellos servicios que conllevan al cumplimiento de la misión son la esencia dela biblioteca. Su realización no requiere de costos adicionales, ni de infraestructura especial y se ofrecen en forma permanente. En esta categoría se ubican los servicios de consulta, préstamo y referencia.

Consulta: Acceso directo del usuario a las colecciones y a su uso en el interior de la biblioteca.

Referencia: Es la guía y orientación a los usuarios en la búsqueda, localización y obtención de información. Este servicio puede realizarse en forma personal, telefónica o por correspondencia postal o electrónica.

* Préstamo: Suministro de materiales bibliográficos para ser consultados en espacios diferentes al de la biblioteca, incluye la modalidad de préstamo interbibliotecario.

5 Irma Isaza R., ClemenciaMolinaE. y MarthaAlicia Pérez G. Serviaosdeinfomaciónenbibidtecasescda resypúblicas Ameria: Universidad del Quindío, 1991. p. 42. 


\section{Servicios complementarios}

Son aquellos cuyo diseño y realización requieren de un costo adicional y de una infraestructura especial. Su prestación o realización se define en tiempos específicos. El diseño de estos servicios esta condicionado y directamente relacionado con las capacidades financieras y de gestión que tenga la biblioteca, algunos de éstos son: alerta, elaboración de bibliografías, servicios de extensión, etcétera.

Para el diseño de servicios y programas es indispensable que la biblioteca cuente con colecciones, independientemente del soporte y del formato en el cual se encuentren éstas. Su selección, organización y mantenimiento ofrecen una visión de las dinámicas de la biblioteca. "La colección de una biblioteca es un organismo vivo y debe, por lo tanto, cambiar continuamente como cambian los objetivos que la orientan, las técnicas de organización y los usuarios que la caracterizan" . ${ }^{6}$

Los servicios, programas y colecciones existen o se llevan a cabo para satisfacer las necesidades de información, cultura, educación y de uso de tiempo libre de los usuarios de la biblioteca, razón por la cual es necesario pensar en el usuario como el componente fundamental de toda unidad de información ya que constituye su razón de ser; es por él que la biblioteca tiene sentido. A su vez para que exista el usuario se requiere de la ofertade un producto o servicio. En el caso de la biblioteca este producto es la información, a partir de la cual se generan servicios mediados por procesos decomunicación. "el usuario es el personaje principal de la trama informativa, es el principio y fin del ciclo de transferencia de la información: él solicita, analiza, evalúa y recrea la información". ${ }^{7}$

\section{U suarios}

Los usuarios son aquellas personas para quienes están diseñados los productos 0 servicios de la biblioteca. Se pueden clasificar en usuarios reales y potenciales.

Usuarioreal: persona que utiliza los servicios y programas de la biblioteca en forma frecuente.

Usuario potenial: persona que pertenece al radio de acción de la biblioteca y no hace uso de los servicios y programas, pero podría hacerlo en algún momento.

En la gráfica de la página siguiente se puedeapreciar el uso que hacen de la biblioteca los jóvenes.

D entro de los usuarios de la biblioteca pública popular sólo se tiene en cuenta a los jóvenes por ser éstos los actores de la investigación, para lo cual se estructuró la siguiente categoría de usuarios:

6 MarthaA Alicia Pérez G y TeresitaMondragón Jaramillo. Pdíticaspara d desamdlodecolecionesdd Siste ma deBiblicteras dela UnivesidaddeAntioquia Medellín: E.I.B., 2000. p. XXXIX, 1.

7 Patricia Hernández S. El perfil del usuario de información. En : InvestigaaónBiblideedógica Vol. 7, no. $15 ;$ p. 16-22. 
* Jóvenes escolarizados usuarios (usuarios reales)

* Jóvenes escolarizados no usuarios (usuarios potenciales)

* Jóvenes desescolarizados usuarios (usuarios reales)

* Jóvenes desescolarizados no usuarios (usuarios potenciales)

* Jóvenes colaboradores o proponentes (usuarios reales)

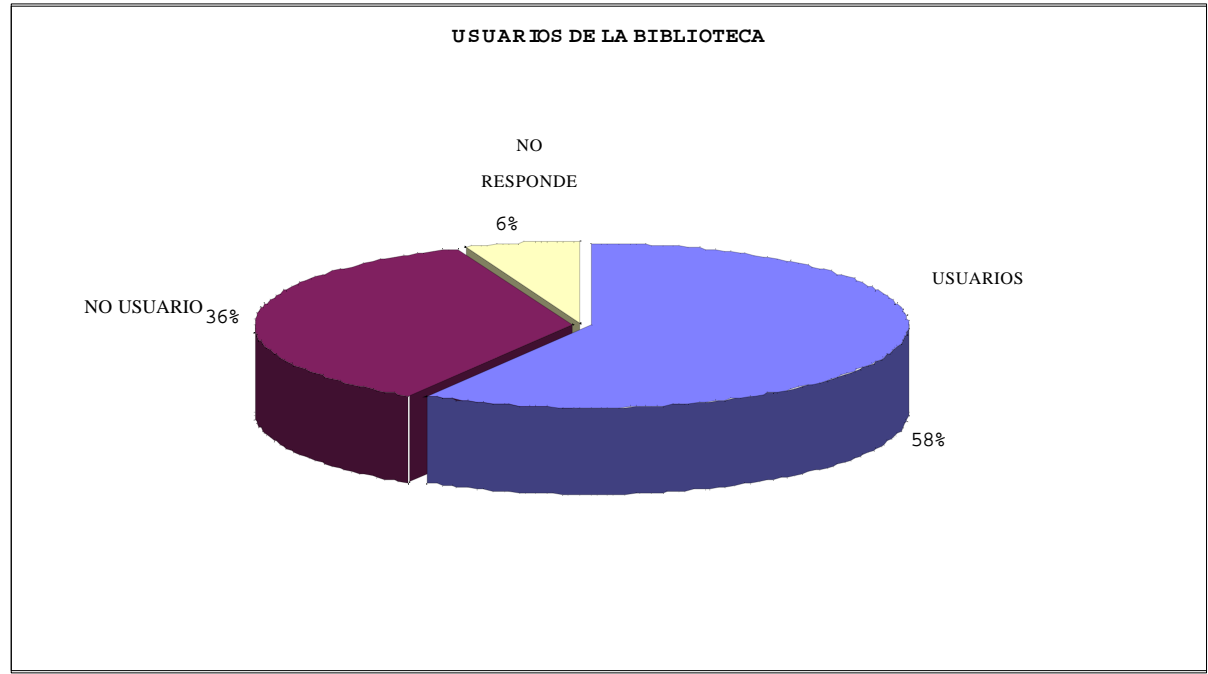

Los usuarios reales corresponden sobre todo a quienes hacen un uso escolar, es decir aquellos que están adscritos al sistema educativo. Este uso lo determinan básicamente: la deficiencia en las colecciones; la formación y capacitación de quienes coordinan la biblioteca; el diseño de programas y servicios para otros segmentos de la población; los horarios, (que son breves) de prestación de los servicios, y los espacios y mobiliario con el que cuente la biblioteca. Como testimonios se retoman los siguientes:" "Básicamenteasisten estudiantes debadilleratoquevana buscar las tareas". "Se mantienenmudholasmuchadhasquecstudian comerio". "Losjónenesdesesclanizadosconsideran queesteespacionoes para ellos, quees sdamentepara los estudiantes".

También se encontró la categoría de usuario proponente o colaborador. En su mayoría se trata de jóvenes que realizan el servicio social del estudiante en la biblioteca, o de líderes que conforman los gruposjuveniles o grupos pro-bibliotecas. "Hay getequeen realidadsecompromte, los dabaradares han salidbcombalfabdizadares, leshagus tadb d trabajo en la biblicteea y sehan quectadb trabajandbcan nostros".

8 Como producto del rastreo de fuentes primarias se incluyen algunos testimonios en letra cursiva entrecomillados que a partir de aquí se asentarán. 


\section{Jóvenes}

\begin{abstract}
Mudhadhasymudhadhos ddtados dereursos materiales yculturales my variados segúnla posicón soial desusfamiliasyd lugar donde vivenyestán expuestos deforma my desigual a los niegos dela videnia, las drocas, la gue na, ed desempleoyla miseia. Losjóenes simbdizan æemundonuevoquenodomina mos, ayos contamos noconocmos bien Michéle Petit.
\end{abstract}

Considerar a los jóvenes como sujetos y actores principales en la búsqueda en ellos de la incidencia de la biblioteca, propició una descripción y análisis de los aspectos que están estrechamente relacionados con este sector poblacional : estéticos, lingüísticos de agrupaciones y prácticas cotidianas; condicionados por factores relacionados con las generaciones; de moratoria social y de género y clase social (aspectosy factores que son ampliamente desarrollados en la investigación). No es posible considerar a los jóvenes fuera del contexto en el que se mueven y de sus características propias, las cuales se constituyen en factores inherentes a esa etapa de la vida en la que suceden los cambios más transcendentales no sólo en lo físico sino también en lo psicosocial. En esa etapa generalmente la persona trata de establecer su identidad, fortalecer su personalidad y constituirse como sujeto activo dentro de la sociedad. Este proceso de búsqueda de identidad y de socialización es determinado básicamente por condiciones sociales, políticas, culturales, educativas y económicas, razón por la cual se construyó un concepto que se articulara con los elementos requeridos para describir dicho sector poblacional: "Los jóvenes son una construcción histórica, política, social y cultural, enmarcada en una cronología de 15 - 24 años de edad, que se caracterizan por tener lenguajes, expresiones estéticas, formas de agruparse y prácticas cotidianas determinadas por la generación, la moratoria, el género y la clase social, a partir de las cuales buscan establecer la identidad y fortalecer la personalidad". 9

Es con base en este concepto como la investigación desarrolló la noción de incidencia, tomando en cuenta la participación y toma de decisiones de los jóvenes en aspectos relacionados con su proyecto de viday el desarrollo de su comunidad, todo lo cual se articula con la Constitución Política de Colombia, que plantea lanecesidad de que las personas participen en las decisiones que afectan su vida económica, social y política. Lo anterior sólo es posiblesi sereconoce alainformación como requisito indispensable para la participación, y a la biblioteca pública como una de las ins-

9 O rlanda Jaramillo đal. Inidaniadelabiblidteca pública enlosjóenesde15- 24añosdeecladdela Comma Sés, Zona Norocidatal deMeddlín Medellín : Escuela Interamericana de Bibliotecología, 1999. p. 160. 
tituciones que posibilitan el acceso libre a la información y al conocimiento que contribuyen al desarrollo comunitario.

\section{INCIDENCIA DE LABIBLIOTECA}

Cuandbsevieenlossuburbios etá uno destinadba tenermalos etudios, a tener un trabajoasqueroso Hayuna gran cantidad deaconteimientos quelohacenir a unoendeta dreción Yosupesquivar eso, conetime enanticonformista, immepor crolado, ahí etá mi lugar[...Los 'nudos'] hacenloquela soieclad espara quehaganyya sonvidentos, sonvilgares, sonimaltos Dicen 'Yovivenlos suburios, entonessoyas', yyoya fui comodlos El hethodetener biblictezascomoéta mepemitióentrar allí, verir, conocer ctras gentes Unabibictea sirvepara «o[...] Yoøog mi vidayellosma

\section{Michéle Petit}

Considerando la incidencia delabiblioteca como esa huella o marca que queda en los jóvenes a partir de su uso, la investigación indagó sobre las relaciones y vínculos establecidos entre los programas y servicios que desarrolla la biblioteca pública popular y los jóvenes. Por lo tanto fue preciso profundizar sobre el uso que hacen de los servicios y programas de la bibliotecalos jóvenes, sobre la satisfacción de susnecesidades de información en el ámbito educativo, recreativo, cultural y organizativo, y sobre el grado de satisfacción que estos usos generan.

Para determinar la huella de la biblioteca pública popular en un sector específico de la población, como es el de los jóvenes, se partió del reconocimiento de las relaciones y vínculos que se tejen entre unos y otros, y que se establecen tanto a partir de los servicios, programas, mecanismos de comunicación y de convocatoria que la biblioteca ofrece a esta población, como de la respuestay demanda que éstos le expresan a la institución. Para lograr una descripción más precisa, reflejo de la realidad, se partió del análisis de los usos que hacen los jóvenes de la biblioteca en lo educativo, lo recreativo (tiempo libre y acceso a las manifestaciones culturales), lo informativo y lo organizativo.

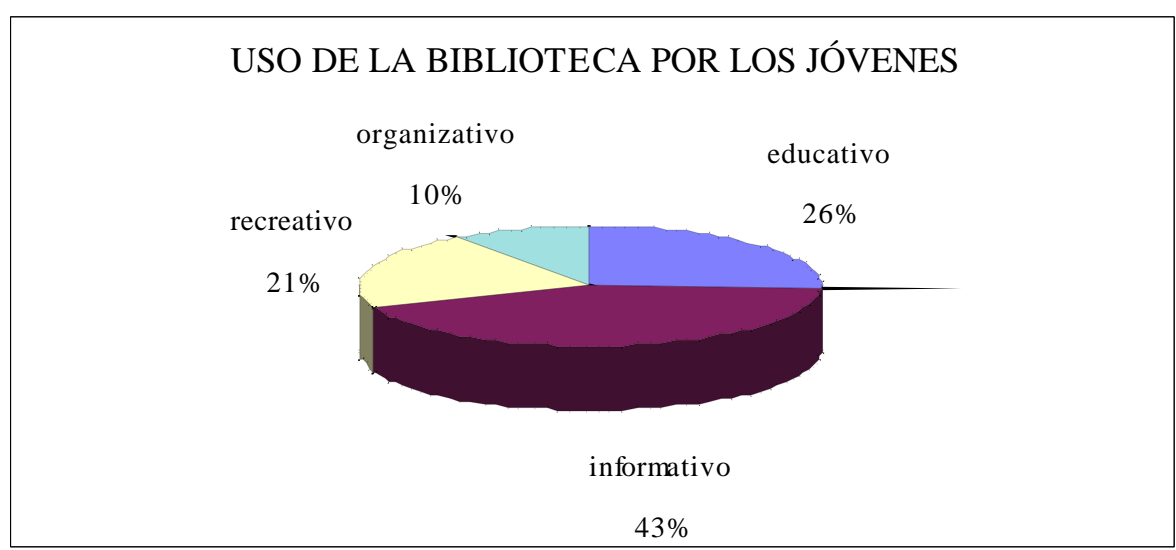




\section{U so educativo}

\section{Como respuesta a las tareas escolares}

Se da un uso escolar cuando la biblioteca ayuda a solucionar las necesidades de información reguladas por el sistema educativo, es decir, complementa los requerimientos de la escuela y responde así a una dinámica propia de la educación formal. Losjóvenes escolarizados usan la biblioteca pública popular más para dar respuesta a las preguntas que les formulan los docentes que a las preguntas que les surgen de sus necesidades e intereses por conocer el mundo.

En general, las consultas de los jóvenes escolarizados son repetitivas y el resultado es una demanda homogénea de información. Frente a esta demanda, bibliotecarios y alfabetizadores expresan: "Lascdecionesamplensufuncóndeayudararęlizarlas tareasdelos esdares Las clecionesen general, sduionanlas neecidades deinfammaióndelos usuarios, induyentodbslostemas esclares". Este uso escolar se refleja en un usuario ocasional o esporádico, que sólo va a resolver la tarea.

Lo anterior es ratificado por el bibliotecólogo brasileño Luis Milanessi, cuando plantea: "Los usuarios delasbibicteras públicas las frementan máspor un deberimpursto, que porunaneecsidad"10 y por la bibliotecóloga G loria María Rodríguez: "Paranadieesunse atoy hay estudiosquelocamdbaran, qued usuaniohabitual, ennuestromedio, aandealasbiblio tecaspúblicas con e propósitofundamental deresdver consultas relacionadas conla eeducacoónformal ; aproximadamented 80\% delos usuarios san estudiantes, su damanda deinformacón es acadḱmica yutilizanla biblicteca comounlugar de€ududio". ${ }^{11}$ Y también por los siguientes testimonios : "Loquemássemueenlabiblidecasonlostemasescdares, yolleøgalabiblicteca y hay un cerodelibros para arganizar, toobs san temas esclares". En la gráfica se pueden apreciar los factores que motivan a los jóvenes a usar la biblioteca.

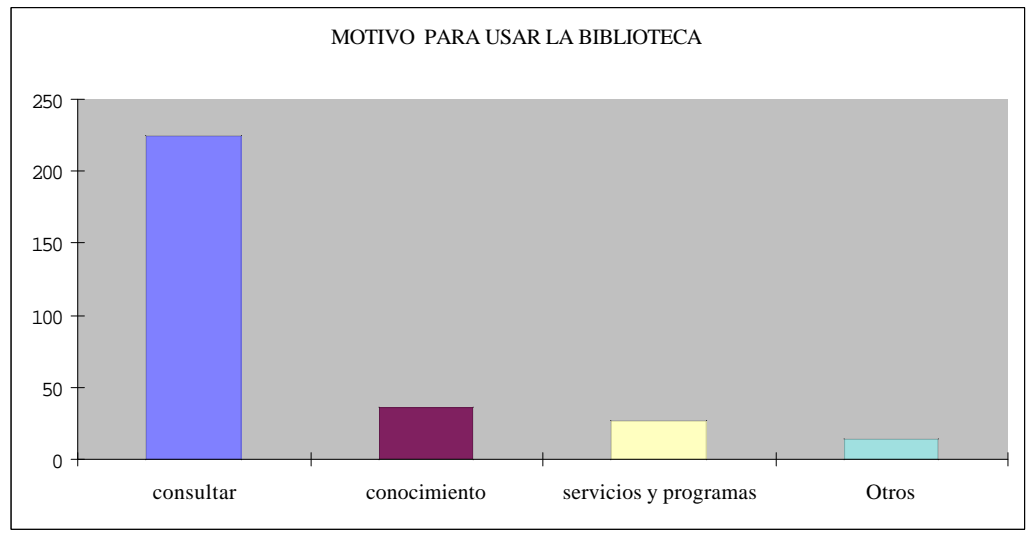

10 Luis Milanesi. Palancas de lectura. En SegundoCongesoNadinal deLectura Santaféde Bogotá : Fundalectura (Mayo 4 de 1995) ; p.3.

11 Gloria María Rodríguez S. Foro sobre bibliotecas públicas. En CongesoNaaional deBiblidtedoǵa Medellín. Ascolbi. (julio 23-25 1998) ; p. 4. 
La alta demanda de la biblioteca por parte de los escolares incide en la alta rotación de los libros de texto y las obras de referencia, lo que de alguna manera deja fuera de circulación a otros materiales existentes en la biblioteca: "Loquemásconsiltanlos usuarios, esliteratura, españd, sociales, geografíaunivesal, temascominformáticayrdigón". El 89\% de los jóvenes encuestados usan la biblioteca para resolver las tareas escolares: "Nosllegand mismodia a preguntar losmismostemas, sanlosmismoscampañers, unosconoen másparahacer la tarea, perosevequees a mismotemarequeidopor d clegio, mañama vienectro joven a preguntar e mismotema".

Las anteriores expresiones constatan que un alto porcentaje de las consultas que hacen los jóvenes responde más a los requerimientos escolares que a los intereses y dinámicas propias de ellos, de ahí que regularmente sean repetitivas y se presenten en los periodos académicos, situación no extraña debido a que la biblioteca pública popular, desde su surgimiento, orienta su quehacer hacia el cumplimiento del objetivo educativo, pues el apoyo a la educación formal aparece como una de las necesidades más inmediatas por resolver. En el uso escolar de la biblioteca es posible entonces identificar los siguientes factores:

* Carencia de bibliotecas escolares, lo cual se manifiesta en dos sentidos: centros educativos que no cuentan con biblioteca escolar que soportey apoye el proceso de enseñanza - aprendizaje, y centros educativos que cuentan con biblioteca escolar y cuyos servicios y programas están orientados exclusivamente a satisfacer a la comunidad académica adscrita a la institución y con horarios paralelos a la jornada académica.

* Condiciones socioeconómicas. La adquisición de materiales educativos y de información es poco usual en los hogares de estos sectores, en ocasiones de bido a factores de orden estrictamente económico (capacidad adquisitiva para invertir en libros) y en otras a factores culturales (no existe el hábito de compra de libros e información). Estos factores hacen que los jóvenes escolarizados busquen en la biblioteca pública popular la respuesta a sus tareas escolares, como complemento a los procesos educativos. D entro del factor socioeconómico, cabe resaltar que la existencia de la biblioteca pública popular evita que los jóvenes se desplacen a las bibliotecas del centro de la ciudad, lo cual representa mayores costos y tiempo de desplazamiento, tal como se puede observar en estos testimonios: "La neesidad deuna bibictea publica ppular en d bamioesmásquelosniñostengan quetradadarseexpuestosal pediga Labibidteca de bamiomeeitabaira la Biblictea Pública Pildo enlabiblictea dd bamiomeatendian conmuhocariño, la palabrasilenioexistíaporquelahabían cdgadb, mohabía unfunainario queestuviera pidiendb silenio".

\section{Para realizar el servicio social del estudiante}

O tros jóvenes manifestaron un uso de la biblioteca pública popular para realizar el "Servicio Social del Estudiante" (programa del sistema educativo, que consiste en que todo estudiante matriculado en el grado 10 u 11, debe dedicar 120 horas a una 
actividad social). Este programa es denominado por los estudiantes como "alfabetizar". Los bibliotecarios, frente a la biblioteca como espacio para la alfabetización, expresan: "Ellosvienenysiemprepreguntansi puedenalfabetizaryquéserequiere El requisito quelesexig்mosesquequieranalabiblicteraylesgusted trabajoconloslibrosyconlagnte'.

La biblioteca se convierte así en institución cooperante con el sistema educativo y con las instituciones educativas, lo que le garantiza reconocimiento y estatus en las comunidades. "Unagranventajaquetienelabiblideea eslaayudadelosalfabdizadbres, comolabibicteca está integradaal NúdeodeV ida Ciudadana, nos rodkan varios đlejos GuillemoCano Isaza, RicardoRendónBravo, laEscudaEl Rosal, CdegjodeSanJudas, Manud JoséGóme, AlfreebCok; todbsestos dejejosnosfailitanuna deta pemanentedealfabedizadores, sonuna ayuda indudablemente, parquea unolequeda my grandeatendar tanta podlacón estudiantil”.

En el proceso de alfabetización en la biblioteca pública popular los jóvenes tienen la posibilidad de ser actores sociales y ejercer roles de liderazgo y compromiso con la comunidad, además de adquirir una formación y capacitación en quehaceres que posteriormente serán útiles en su desempeño laboral. Tal como lo confirman las siguientes expresiones: "Enlabibicterasesensibilizaalosalfabetizadoresyencietameida seles brinda una capacitación, buscandoquedlos, en easnoventa horas, haganumabuena amis tad, serespeten yestablezcan lazos deconivenaia. Comotenemos un talleritodeenarademacoón

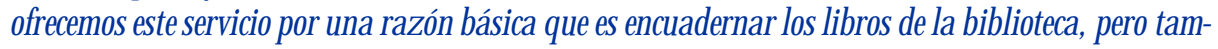
biénbuscandbquedlosaprendanyquecstolespuedaservirmásaddante, ya sea para amejarsus propioslibrosocamofuentedeemple”. "El papd di alfabdizadorescamod deunprofesor, esher cr qued usuariosesienta en confianza enla biblicteea, nocomoun extraña quedialoguecon 8 usuarioy trabajeconla gentey la commidad".

Además de los aportes anteriores, los jóvenes tienen la oportunidad de apropiarse de información y conocimiento, y de capacitarse en el uso y manejo de la biblioteca, situación que en ocasiones los motiva a elegir la bibliotecología como profesión: "Porgjemplohaymuhagentequealfabđizóenlabibliđteca y está estudiandbbiblicteedoǵa, entoneesumoś vequelaalfabđtizacón enlabiblictecalesirvió". "Losjóenesquehanalfabtizadb estándentrodelosprocsosquehayenlabiblicteca, drosestánenguposdedarza, mísica, teatro. Unopiensaqueendietamedida, labiblicteahaservidbparaquelosjóenessevinailenaadividades quetienen quever conla ailturayla educacoón".

O tro elemento quemerece destacarse es la característica especial de hogar que ellos le dan a la biblioteca, lo que regularmente hace que se conviertan en usuarios activos, que conocen y disfrutan de ese espacio: "Cuandbteriaqueirlabiblictea, lohacia parapro yectamey estar ahí, ahora disfruto di espacioy pureb queckmey ser la ultima en salir".

Ese "quedarme" se puede interpretar como una ampliación del espacio vital o "segundo hogar", que los jóvenes le asignan a la biblioteca. Ya son de ahí, pertenecen ahí, el espacio es suyo porque se lo han apropiado. Es un espacio queles permite satisfacer sus requerimientos académicos, hacer las tareas y alfabetizar. Es por esta razón que algunos lo convierten en espacio vital. Hay jóvenes que luego de entrar a la biblioteca como alfabetizadores tienen la posibilidad de dar un giro y convertirla en un lugar de trabajo, de encuentro, de crecimiento personal que les permite resolver 
dudas, conocerla, saber lo que hay y lo que no hay, estar en contacto permanentecon los usuarios, y terminan sintiendo tanto gusto con ella que la consideran como una opción de vida y la cargan con la connotación de punto de encuentro, debido a que les permite establecer contactos, conversar, integrarse y establecervínculos deamistad y solidaridad entre ellos. Las anteriores afirmaciones se confirman con la siguiente expresión: "Deprontounohablaahora delabiblictea, demi segundohogar, mi casa, parqueumoetáenlabiblideea, paqueyallevadíastrasdíasenlabibidicea, conesecontadoconla gente, perohayqueirlabiblicteea, puesescomod segundbhogaroesmudhomás'. Esta metáfora muestra cómo aflora en los jóvenes alfabetizadores el sentido de pertenencia, de gusto y de amor por la biblio teca: "Nostroshemosaprendidbateneleamralabiblideea”.

\section{U so recreativo}

Se habla de uso recreativo en la biblioteca pública popular cuando los jóvenes la identifican como un espacio preferido para ocupar su tiempo libre, para encontrarse con el otro o para participar en actividades de carácter lúdico o cultural. Dicho uso responde a un acto libre, voluntario y está directamente relacionado con los intereses, motivaciones y gustos de los jóvenes. Es ese rasgo precisamente el que muestra la capacidad creativa de la biblioteca para convocarlos y donde los servicios de extensión bibliotecarios son el eje dinamizador de múltiples opciones paralos jóvenes.

Por consiguiente este uso se plantea como fundamental para que la biblioteca cumpla su función social; al respecto Lucila Martínez de Cáceres se refiere a la biblioteca como: "Una puerta de acceso a emociones, respuestas, soluciones, experiencias gratificantes y encuentro del placer que da la posibilidad de volar con la imaginación, de crear y tener nuevas ideas, de solucionar problemas sencillos y complejos". 12

La Biblioteca pública popular es un espacio de acogida para los jóvenes, donde no se establecen requisitos ni condiciones para su uso y donde se les brinda, independientemente de sus condiciones educativas y sociales, de su hacer o no hacer en la sociedad, un espacio de confianzay una posibilidad más para hacer uso de su tiempo libre. Es así como la biblioteca busca "animar procesos que favorezcan la expresión y que constituyan formas de iniciación o desarrollo de lenguajes creativos y de la capacidad de innovación y búsqueda de nuevas formas y expresiones". ${ }^{13} \mathrm{D}$ e esta manera la biblioteca pública popular, como institución de carácter social y cultural, desempeña un papel importante en el uso que las personas hacen de su tiempo libre disponible, entendiendo éste como aquella parte del tiempo, fuera del trabajo y del estudio, destinada al desarrollo físico, emocional e intelectual del ser humano; el tiempo que se dedica al descanso, a la recreación y a la cultura, como se aprecia en

12 Lucila Mártinez de C. Markéingaultural ybiblidtea pública: @trategjasdegrenciacreativa Bogotá : Colcultura, 1991; p.18.

13 Álvaro Agudo, Mirian Mejía .Letura, biblideeaycommidadtresestudios Caracas: IABN, 1993; p. 19. 
este testimonio: 'Yodińaquedlosvanallí con e findeestar unrato, deprontodeaidarseode irserdacionando conla misma persona dela biblicteea".

Lo anterior ratifica una de las características de la biblioteca pública popular: estar abierta a todas las personas, posibilitar las relaciones y brindar opciones en el uso del tiempo libre: "Haygentequenovaallásdamenteainvestigar, simoquearandbestánabumidos, sesientanaleerunlibro Haymuhas histarias para quedlosleany hay gentequeva realmentea leer, a distensionarsedelascosas queleshan pasadben d dia. Iradharlarunrato". Por lo anterior, la biblioteca pública popular se constituye en un espacio para el disfrute de actividades lúdicas, culturales, de formación y de capacitación. En este sentido, los bibliotecarios expresan: "Porġemploalamíavanajugar conlosmaterialesdidádicos, tanbién lesencantaira decrarla, la decoramosen didienbre, cadaépocala decoramosmybonita". En el espacio de la biblioteca, los jóvenes pueden encontrarse con el otro, lo que les permite construir lazos sociales. Es un punto de encuentro en la medida en que se posibilitan laintegración y la socialización: "Participandeloqueesteatro, lesatraemudholoma nual, y hacer pesdores con desehos de materiales es comb alimentar e espíitu”. E ste uso recreativo que hacen los jóvenes de la biblioteca pública popular, la convierte en un espacio vital y de refugio que les permite descansar, aislarse del entorno, distensionarse, hacer productivo el tiempo libre, el cual, según las encuestas realizadas entre los jóvenes del sector va de tres a ocho horas por día.

\section{U so organizativo}

La organización juvenil se entiende como un proceso de construcción colectiva, en el que los jóvenes se proponen alcanzar ideales comunes que apuntan a la construcción ciudadana. La capacidad y disposición para organizarse desempeña un papel importante donde las necesidades, motivaciones e intereses, determinan la conformación de grupos bien sea artísticos, deportivos, académicos, ecológicos 0 religiosos. Estas bibliotecas no están al margen de contribuir a la generación y consolidación de procesos de organización de losjóvenes; por el contrario, en ellas, además de la información que se obtiene, también se da la posibilidad de que se apropien del espacio, el cual puede convertirse en lugar de acogida para reunirse, para compartir experiencias, ideales, visiones y apreciaciones sobre la comunidad, el entorno y la vida misma. D e esta manera la biblioteca pública popular es vista por los jóvenes como un espacio donde es posible realizar actividades de carácter académico, cultural y recreativo, como un lugar de encuentro y trabajo para los grupos organizadosy como sitio donde existen oportunidades para que surjan nuevas dinámicas en las comunidades.

Las siguientes expresiones recogen diversos momentos en los que la biblioteca hajugado un papel importante en la organización de los jóvenes: "Alreekedordelabiblidecaseenauntranlosguposdedanza, deteetro, demúsica, para ensayar, para hecerpresentar dones; tambiénsehacen proyeciones deplíallas(...) entones seda comoun pretextopara quelos beriosseempieena orcganizarymásadłantesecreencompradiones Enlabiblideca encontréuna 
dinámicamyparticipativa, estaban en æemmentomuchosguposqueseorganizabanalleedectr dedla, eneseentoneslossetor sindical, textil,(...) sedabanataenlabiblideea. Enlabibidteea popular empeéa compartirlasleeturas con compañeros da bamio, parqueterían ungupojuveril y drosguposqueseibanformandb La dinámica delabiblideca convocabaalosjóvenesarganiza dosynoarganizadosdelaZana, eanjórenesconinteresespeciales, teníanalgúntipodeproyeción enlabiblideea parala commidad.. Esolosmotivóarealizarctrasadividades, comogruposjuxe niles, muhosjóvenessonloscordinadoresdelosguposjuveniles, a mí mepareequeinfluyen mr dho...) com esas ganas detrabajar, desalir addante, deconocer gentenueva quetienealgp que apartar".

\section{U so informativo}

Se considera como uso informativo de la biblioteca, aquel que el joven hace de manera libre y espontánea, bien sea para ahondar sobre aspectos de su interés y que le permitan variar su nivel de incertidumbre, para adquirir nuevos conocimientos 0 como base para resolver problemas cotidianos.

La biblioteca pública popular es el espacio donde la persona busca respuesta a sus necesidades de información, ya que por medio de preguntas expresa su necesidad de información y sus deseos de conocer.

Puede decirse que la biblioteca es un espacio para las preguntas, un espacio donde tiene cabida el desconocimiento. La biblioteca acepta que el ser humano pueda desconocer y brindainformación, no cuestiona el desconocimiento, es un lugar dela sociedad donde se acoge justamente esa debilidad de no saber, a partir de la apertura que se da para preguntar. Es un espacio de acogida para el débil, para el que está en condiciones de inferioridad, de desventaja. Pero la biblioteca como espacio para hacer preguntas no es unidimensional: tanto el usuario como el bibliotecario seformulan preguntas y a través de éstas se establecen puentes de comunicación e interacción entre el bibliotecario y los jóvenes.

Para cumplir con ello la biblioteca diseña servicios y programas, y desarrolla sus colecciones, las cuales suelen presentar variedad de soportes y temáticas. Lo que se propone es cumplir con los objetivos y las demandas actuales de los jóvenes dentro de marcos de calidad, actualidad y pertinencia.

\section{A propiación de información}

La apropiación de información por parte de los jóvenes en la biblioteca pública popular se da con base en tres variables básicas. La primera la constituye un conocimiento del espacio, la segunda está dada por la posibilidad de tener una formación que facilite el uso y manejo de la biblioteca y de sus recursos, y la tercera la determina el uso continuado y permanente, a partir del cual se reconoce la lógica organizacional, la información y las propias necesidades de información. Es así como surge el usuario activo, el cual se configura a partir de un contacto con la biblioteca y de un conocimiento de sus recursos mediado por el gusto y el interés de informarse. 
Laapropiación del espacio precedeala apropiación delainformación: conocer el espacio de la biblioteca, moverse en él, constituye un requisito para lograr la apropiación de la información.

El usuario activo, que en algunos casos se convierte en colaborador o proponente, establece con la biblioteca un contacto significativo, cualitativamente diferencial y un conocimiento preferencial. Los jóvenes hacen uso de la biblioteca y la reconocen como un espacio para informarse cuando dicen: "Labiblideea esfuentedeinfommdón, dalaqpartunidaddeabrirseun espacio, parqueunomismoarandbquieeinteresarsepor una cosa, vaalabiblidtecaylee Yosoydelas pessanasquecadavez quesaleunlibroquemepareebue nolollewoen préstam, ettances es cambesa, combcanoer, combtener ganas deinformarte".

La apropiación de información está directamente relacionada con la forma, temática y soportes que conlleva la información. En lo relativo a la forma (el cómo se presenta la información) se aprecia que el gusto de los jóvenes está más orientado a la literatura "liviana". Es así como a partir de los diversos instrumentos de recolección de información de la investigación, se conocieron las preferencias de lectura e información de los jóvenes: “...A mí megusta murholosenillo, lofáil yloresumidb".

Esta preferencia de información de los jóvenes se relaciona en gran medida con procesos de lectura que se desarrollan en la educación y tiende más a responder a problemas planteados por la escuela, que aquellos que son propios de su entorno, sus intereses y sus necesidades, en los que la lectura recreativa no tiene una significativa presencia en las prácticas cotidianas.

Por lo que toca a las temáticas se encontró que lo más preferido por los jóvenes está relacionado con los temas que son propios de su edad y de su entorno, lo que corresponde a sus gustos, intereses y motivaciones, aquellos que de alguna manera reflejan sus prácticas cotidianas: temas de actualidad, farándula, deporte, sexualidad, drogadicción, ciencia - ficción, esoterismo y valores: "A mi megustaleerlibrosdevalores porquemepareequesoloneesitaunosiemprepara todalavida, para ceer dianiamenteenvalores, parasaber trataralagente, parquesiempremepareenmyimpatantes". No obstante haber encontrado una preferencia por la literatura liviana, existen algunos jóvenes que destacan la lectura de cuentos, novelas, poesía y frases célebres.

Es importante resaltar que los jóvenes de hoy están más inclinados por la imagen, el audio, la multimedia, lo virtual; es decir, todo aquello que les represente velocidad, vértigo, simultaneidad e interactividad, características propias de las tecnologías modernas de comunicación e información. A partir de esto tienen la posibilidad de interrelacionarse con el desarrollo tecnológico, del cual necesitan tener una visión, y aplicarlo asu entorno, para entrar a un mundo cadavez más competitivo y queavanza vertiginosamente.

Las bibliotecas públicas populares no cuentan con tecnologías modernas de información, situación que limita la búsqueday apropiación de la información actualizada y atractiva. D e ahí que sea necesario incluir en sus colecciones documentos en diferentes soportes que cubran todas las áreas del conocimiento a fin de satisfacer los gustos de los lectores, sea cual fuere su grado de escolaridad. Es más fácil atraer a 
los jóvenes para que hagan uso de la biblioteca si se incorporan nuevos soportes de información, debido a que este sector de la población, es el más motivado a aceptar las nuevas tecnologías de información y comunicación. Q ue las bibliotecas estuvieran dotadas de estas tecnologías es el mayor deseo expresado por los jóvenes entrevistados y encuestados en la investigación.

\section{A MODO DE SÍNTESIS}

Lainvestigación halló que la incidencia de la biblioteca pública popular en los jóvenes se da fundamentalmente en dos aspectos:

* El primero lo constituye la realización del objetivo esencial para el cual fueron creadas las bibliotecas públicas populares: contribuir alos procesos educativos y culturales de la comunidad; lo que se basa en el apoyo que se le da a las actividades escolares y el ofrecimiento que hace la biblioteca para que los jóvenes realicen el Servicio Social del Estudiante. Además, esta incidencia en lo escolar tiene consecuencias directas con la situación económica, debido a que permite el ahorro y por tanto la adquisición de materiales educativos e informativos, y facilita el desplazamiento a otras bibliotecas de la ciudad; incidencia que es más significativa y se marca con mayor énfasis en los jóvenes que hacen la alfabetización en la biblioteca, pues en ella descubren, interpretan y gustan de la información de acuerdo con sus capacidades, habilidades y destrezas. De esta manera, la biblioteca contribuye a la recomponer la identidad, a partir del sano disfrute del tiempo libre, de la lectura, dela realización de las actividades extra escolaresy del apoyo a las organizaciones o agrupaciones juveniles.

* El segundo aspecto está relacionado con la biblioteca como espacio vital. A partir de los servicios y programas, la biblioteca les brinda a los jóvenes la posibilidad de habitar, crear, recrear y disfrutar de su espacio.

El elemento común en ambos aspectos eslautilización del espacio, yeso posibilitala convivencia, el confort, la comodidad; eso reivindica y dignifica su condición de serjóvenes; eso valora su ser, sus actividades, aptitudes, conocimientos y desconocimientos. D e ahí que la biblioteca sea considerada como un lugar donde es posible la creación y el esparcimiento, en el que se le permite a los jóvenes enfrentar su devenir, y comprender y reorientar su autonomía. Un lugar que les permita buscar un horizonte que les ayude a contemplar la vida desde una visión diferente ala que dala esquina, la calle, o la vida estrecha del barrio y del entorno; que les permita crear vínculos con los libros, el bibliotecario y otros usuarios; y que les ayude a establecer pequeñas redes por las que comiencen a circular sensibilidades relacionados con la información, la organización, la solidaridad y laintegración con la comunidad. Todo esto puede constatarse en las siguientes expresiones: "Si, mevi dudadano di mmob arandbmedi aunta enloslibros quehabía ctras cosas, yohabía aguantadbhambreconmi gente, 
peronunca comoenlospasajesdeFeelorDostoyedi, enlosqueal igual d hambrecampea desdela pimerahastalaúltima página. Estardaciónqueyotería conloslibros, conlalectura quefuemi compañía en los problemas queture, en mis soledades, aandomi papáymi mamá sesepararon, arandbllegueaunađiudadcomoBogdá. Haydrostextosquelavidatetraeysan contrastantes, te cogny teestreganla realidadyahí medbycuenta quenosoy e únicoquehatenidbcontrastes, difialtades, hambre".

Se afirma, pues, que la biblioteca pública se constituye en un espacio aglutinador de la comunidad, no sólo en torno a lo educativo sino también en aspectos de primordial importancia que rodean y permiten el desarrollo de la educación: la recreación, la información, la cultura y la organización.

Vale la pena resaltar que no sólo la biblioteca incide en los jóvenes, sino que a su vez éstos inciden en la biblioteca pública popular. Por lo regular estas bibliotecas están bajo la responsabilidad de dicho sector generacional por estar coordinadas por jóvenes líderes de la comunidad, en muchas ocasiones de manera voluntaria. Esta influencia en los jóvenes en perceptible tanto en la selección de las colecciones como en el diseño y la orientación de los servicios.

La biblioteca pública popular es un tipo de biblioteca pública cuyas características propias son determinadas por el contexto, los sujetos y las distintas maneras de interacción. Este tipo de biblioteca se constituye en un escenario importante para losjóvenes que viven su proceso de moratoria, lo que tiene especial relevancia en los procesos por los que ellos transitan: desarrollo, identidad, organización y formación. Esto le otorga a la biblioteca grandes posibilidades para el encuentro, la formación, la capacitación, el esparcimiento y la recreación; un espacio donde tiene cabida el desconocimiento, donde se reconoce al joven por sus potencialidades y donde éste es sujeto clave en la creación, funcionamiento y desarrollo de estas bibliotecas.

La biblioteca pública y la popular enfrentan una época de grandes desafíos, como le ocurre a otras instituciones educativas, y debe redimensionar su papel y buscar posibilidades que le permitan darle más valor agregado a su función social. Para ello requieren conectarse con el mundo de las tecnologías de la información y la comunicación, y actualizarse y comprender lo que está sucediendo en el entorno de lo creativo, en la estética y en las formas de expresión, sentimiento y creación de los jóvenes.

\section{BIBLIOGRAFÍA}

Ag UD o G., Alvaro; Mejía, Myriam. Letura, bibicteeaycommidad: tresestur dios Caracas : IABN; CERLAC. $301 \mathrm{p}$.

ALVAREZ Vélez, William. Situadóndd serviaodeextensiónaltural enlasbibliotecaspúblicasdd V alledeAbura Medellín: Universidad de Antioquia, Escuela Interamericana de Bibliotecología, 1994.. 339 p. (Tesis)

Álvarez Zapata, Didier. "Productividad y misión de la biblioteca pública Latinoamericana" En: Hgias delectura. Bogotá. No. 26 (Feb. 1994); P.10 - 13 . 
BAYLON, Anahi ... [t. al]; IFLA. "Fundamentos y estrategias para integrar la Biblioteca pública y los programas de alfabetización y postalfabetización de América Latina y el Caribe" En: RemiónRegional debildidecas Pút bicas(Piura: Abr. 23-25 1996). Lima: Biblioteca Nacional; Universidad de Piura, $1996.13 \mathrm{~h}$.

CUERVo Castrillón, Ana Cristina; Ríos O sorio, Adela. Bildideca ppulary desamollosodal : ZonaNorocidantal deMeddlín Meddlín: FaaltaddeCienaas SocialesyH Humanas,1997.p. 44. Tesis (E specialista en G erencia del D esarrollo So cial) Universidad de Antioquia, Facultad de Ciencias Sociales y Humanas.

ECHAVARRÍA G., Elsy. Diseñodeuna reed para biblideraspopularesdd MuniapiodeMeddlín Medellín: Escuela de Bibliotecología, 1994. 242 p. Tesis (Bibliotecóloga) Universidad de Antioquia, Escuela Interamericana de Bibliotecología.

FUND ALECTURA. "Encuesta sobre comportamiento e interés de lectura de los Colombianos" En: HgasdeLetura Bogotá. No. 16 (Jun. 1992); p. 14.

HERNÁND EZ S., Patricia. "El perfil del usuario de información" En: InvestigaaónBiblictedógica México. Vol. 7 No. 15 (1993); p. 16-22.

HeRRERA Cortés, Rocío. "Impacto de la biblioteca pública en la satisfacción de las necesidades de bibliotecología". En: ReistaInteramericanade Biblictedoǵa. Medellín. Vol. 16, No. 2 (Jul. - Dic. 1993); p. 85 - 100.

JARAMILLO , O rlanda. Et. al. Inideniadelabiblidecapúblicaenlosjóvenesde15 a24 añosdeeebddelaComma Sés, ZonaNorocidental deMeddlín Meddlín: Esada Interamericana deBiblidtedogóa, 1999.-296 p.

MARTÍNEZ DE C., Lucila. Markeingaltural ybiblideca pública: estrategjasde gerenia cæativa. Bogotá : Colcultura, 1991. 18 p.

MILANESSI, Luis. “Una alternativa al orden” En: HgiasdeLectura Bogotá. No.32 (Feb. 1995); p. 4 - 7.

MILANESSI,. Luis. "Palancas de Lectura”. En: SeeundbCongesoNacional de Lectura(2. : Santafé de Bogotá: Mayo 4 1995);; Santafé de Bogotá: Fundalectura, $1995.10 \mathrm{p}$.

Montoya Ríos, Mónica, Férnandez Pérez, Juan Rafael. Propuesta moddo para creacóndepuntosdeprestacoón deserviaosbiblicterariospúblicos parateminales detranspateterestreyáreas d V alledeAbura. Meedlín: EsadaInterammicana deBiblicteedoóa, 1997. Tesis (Bibliotecólogo). Universidad de Antioquia, Escuela Interamericana de Bibliotecología.

NAVIA V elasco, Carminia. Labibidtecapublicapppular. Cali: Copparacónpara d DesarrdloCultural PopularyCentroCultural Mdéndz, 1989.75 p. 
PÁEz Urdaneta, Iraset. "Bibliotecas públicas: la tercera oleada" En: Reis ta Interameicana de Biblictedoǵa. Medellín. Vol. 15 no.1 (Ene.-Jun. 1992); p. 7-28.

PALMA A., A bsalón. Impadodelasbibidteeaspopularesdelascommas1 y2 dela ZonaNoroiental deMeddlín Meddlín: EscudaInterameicanadeBiblideedo ǵa, 1995. 121 p. Tesis (Bibliotecólogo) Universidad de Antioquia, Escuela Interamericana de Bibliotecología.

PETIT, Michéle. Nuavosacecamietosalosjóvenesyalalectura México : Fondo de Cultura Económica, 199.196 p.

PUBLIC LIBRARY RESEARCH GROUP. "Public Library Aims and Objectives" En: LibraryAssociation Reerd Londres. No. 73 (Dic. 1971); p.3.

RODRÍG UEZ Acevedo, María Consuelo. "Y, la información para qué? : Centros rurales sostenibles de información, una altemativa de paz en tiempos de guerra".Medellín : Escuda Interameicama de Biblidtedogóa Vol. 1., 1998. 211 p. Tesis (Bibliotecóloga) Universidad de Antioquia, Escuela Interamericana de Bibliotecología.

Ro G RíG UEZ S., Gloria María. "Foro de bibliotecas públicas" En: Quinto CongresoNacional deBiblidtedoóa (5. : Medellín : Julio 23-25 1998). Medellín : ASCO LBI, 1998. $11 \mathrm{p}$.

SUAID EN, Emir José. Biblicteca pública einformaçao a commidade Madrid : Sao Paulo: Global, 1995. 112 p.

- - - .Labibicteea públicayd desamdlohumano Brasilia: Universidad de Brasilia. D epartamento de Bibliotecología y Ciencia de la Información, 1997. $16 \mathrm{~h}$.

TOHORDELL, Bird. "Bibliotecas públicas en una sociedad cambiante" En: Hgas deLectura No. 26 (Feb. 1994); p. 16-19.

UNESCO. "Manifiesto de la UNESCO sobre la biblioteca pública”. 1994 En: Hgjas deLeetura Bogotá. No. 35 (Ago. 1995); p. 4-5.

YEPEZ O sorio, Luis Bernardo. Lema y misión social para la Biblioteca Pública en Colombia. Medellín : Comfenalco, 1998. 12 p.

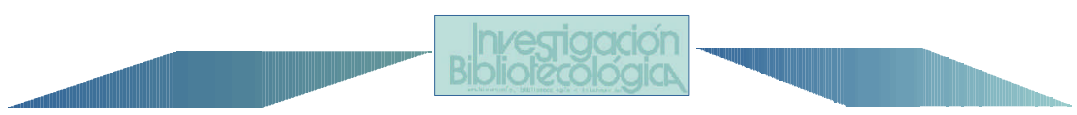

